\title{
Radioactive ion beam manipulation at the IGISOL-4 facility
}

\author{
Heikki Penttilä ${ }^{1, *}$, Olga Beliuskina $^{1}$, Laetitia Canete $^{1}$, Antoine de Roubin ${ }^{1}$, Tommi Eronen ${ }^{1}$, Marjut Hukkanen ${ }^{1}$, Anu \\ Kankainen $^{1}$, Iain Moore ${ }^{1}$, Dmitrii Nesterenko ${ }^{1}$, Philippos Papadakis ${ }^{1,2}$, Ilkka Pohjalainen ${ }^{1}$, Mikael Reponen ${ }^{1}$, Sami Rinta- \\ Antila ${ }^{1}$, Jan Sarén ${ }^{1}$, Juha Uusitalo ${ }^{1}$, Markus Vilén ${ }^{1}$, and Ville Virtanen ${ }^{1}$ \\ ${ }^{1}$ University of Jyvaskyla, Department of Physics, P.O.Box 35 YFL, FI-40014 University of Jyvaskyla, Finland \\ ${ }^{2}$ Oliver Lodge Laboratory, University of Liverpool, Liverpool, UK
}

\begin{abstract}
The IGISOL-4 facility in the JYFL Accelerator Laboratory of the University of Jyvaskyla (JYFLACCLAB) produces low-energy radioactive ion beams, primarily for nuclear spectroscopy, utilizing an ion guide-based, ISOL-type mass separator. Recently, new ion manipulation techniques have been introduced at the IGISOL-4 including the application of the PI-ICR (Phase-Imaging Ion Cyclotron Resonance) technique at the JYFLTRAP Penning trap, as well as commissioning of a Multi-Reflection Time-Of-Flight (MR-TOF) separator/spectrometer. The successful operation of the MR-TOF also required significant improvement of the Radio-Frequency Quadrupole (RFQ) cooler and buncher device beam pulse time structure. In addition, laser ionization techniques have been developed for particular cases, for example, a hot cavity laser ion source for silver production. A new stable isotope ion source and a beam line has been introduced for tuning and calibration purposes. In addition to the installations at the IGISOL-4 facility, the extension of the vacuummode recoil separator MARA (Mass Analysing Recoil Apparatus), MARA-LEB (MARA Low Energy Branch) has been under development. MARA-LEB will utilize the gas-cell technique and laser ionization to convert $\mathrm{MeV}$-scale radioactive beams to low-energy ones.
\end{abstract}

\section{Introduction}

The IGISOL-4 mass separator facility [1] in the JYFL Accelerator Laboratory of the University of Jyvaskyla (JYFL-ACCLAB) produces low-energy radioactive ion beams, primarily for nuclear spectroscopy, utilizing an ion guide-based, ISOL-type mass separator.

The ion-guide technique is based on the survival of recoil ions in noble gas following a nuclear reaction in a thin target. Thus, the mechanism is applicable for all elements, in particular for the refractory ones. Combined with charged particle-induced fission, IGISOL provides a wide selection of neutron-rich beams [2]. A database of mass-separated beam intensities at the electrostatic switchyard (labeled as "5" in Fig 1) located at the focal plane of the IGISOL separator magnet (labeled as "4" in Fig 1) is available for $25-\mathrm{MeV}$ proton-induced fission of natural uranium in connection with the ENSAR2 network CRIBE (Chart of Radioactive Ion Beams in Europe) [3]. A database of proton-induced fission product yields of natural thorium is in preparation.

The use of the ion-guide technique is not limited to fission: neutron-deficient beams can be produced via fusionevaporation reactions with light [4] and heavy ions [5, 6]. Utilisation of multinucleon transfer reactions has been demonstrated [7] as well; further developments towards a transfer reaction ion guide have been in progress recently. In addition, laser ionization techniques have been

*e-mail: penttila@jyu.fi developed to enhance the efficiency of heavy-ion fusionevaporation products.

Although the ion guide source is universal, for particular cases other methods provide better efficiency, which may be essential for the production of some very exotic isotope. As an example, recent developments of a hot cavity laser ion source for silver production [8] will be described in Sect. 3.

The radioactive beams are extracted from the ion guide source into a radio-frequency sextupole ion guide (SPIG) [9] for low-energy transport into a high-vacuum region. The ions are subsequently accelerated to $30 \mathrm{keV}$, mass separated with a self-focussing $55^{\circ}$ dipole magnet, and delivered to an electrostatic beam-switchyard for identification and further experiments.

The resolution $R=M / \Delta M$ of the dipole magnet is of the order of $R \approx 500$, which is sufficient to select a particular mass number $A$. In many spectroscopy experiments being able to choose the isobar $A$ is already adequate. In such cases the beam is delivered without further manipulation to the spectroscopy beam line (labeled as "6" in Fig 1). In the collinear laser experiments ("8" in Fig 1) the presence of the entire isobar $A$ is usually not an issue. However, the time structure and the energy spread of the beam need to be reshaped. The ion beam from the source is continuous (DC), while laser experiments benefit from ions arriving as short pulses in order to optimize the signal-to-background. For laser experiments, the energy spread of the beam should also be minimised. These goals 


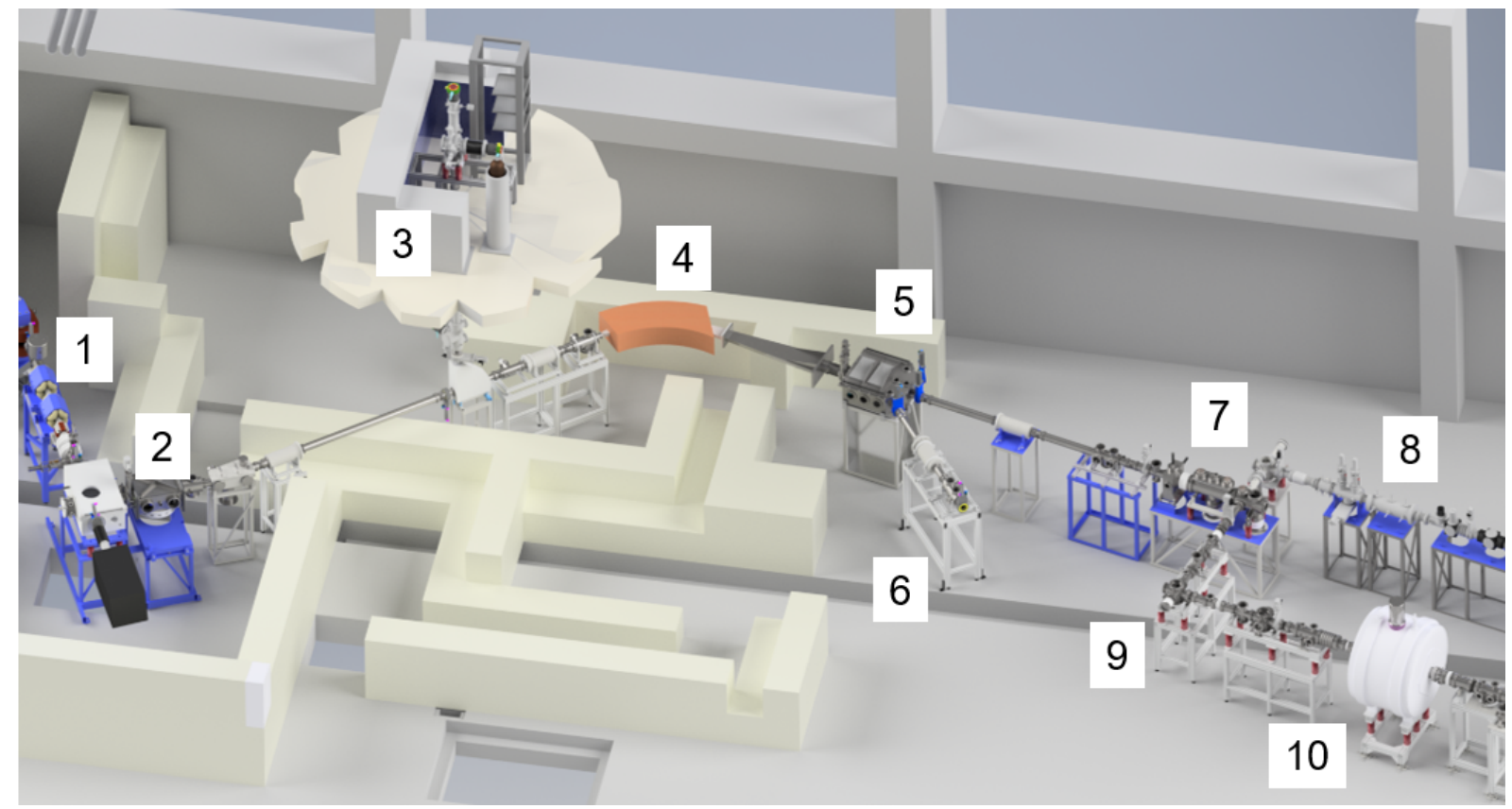

Figure 1. The overview of the IGISOL-4 facility. 1. Primary beam line from the cyclotrons. 2. Target vacuum chamber housing the ion guides/sources. 3. Off-line ion source array. 4. $55^{\circ}$ dipole magnet. 5. Switchyard. 6. Spectroscopy beamline. 7. RFQ cooler and buncher. 8. Laser beamline. 9. Trap beamline. 10. JYFLTRAP. For location of MR-TOF separator, see Fig. 3

are achieved with the radiofrequency quadrupole cooler and buncher, RFQ [10]. It is a linear quadrupole ion trap, where the ions are cooled by allowing them to move in a low pressure helium gas. The ions are trapped by a radiofrequency electric field applied to the quadrupole rods and a DC potential in the trap axis direction. The trapped ions are extracted as a short bunch and directed either to the laser beamline, or to the JYFLTRAP beamline. After the recent modifications of the RFQ, ion pulses can be extracted in two modes. One mode gives very small energy spread $(<1 \mathrm{eV})$ with large temporal width $(\approx 10 \mu \mathrm{s})$ while the other provides short $(<100 \mathrm{~ns})$ with larger $(>5 \mathrm{eV})$ energy spread.

The JYFLTRAP is a double Penning trap installed in a single $7 T$ superconducting magnet. The first, so-called purification trap, is used as a high-resolution mass filter. With it, it is possible to produce mono-isotopic radioactive sources with a mass resolving power $R=M / \Delta M \lesssim 2 \times$ $10^{5}$.

The second Penning trap, called the precision trap, is mostly used for atomic mass measurements but also for advanced ion manipulation techniques such as the Ramsey cleaning technique [12] or the phase-dependent cleaning technique [13], offering a mass filtering with $R>10^{6}$ utilising ion excitations in the precision trap. Such resolution with the Ramsey method allowed for example physical separation of the low-lying $233 \mathrm{keV}$ isomeric state and the ground state of ${ }^{133 m} \mathrm{Xe}[14,15]$. The newly implemented phase-dependent cleaning technique offers even higher mass resolving power. This is shortly discussed in Sect. 5 .

Nevertheless, JYFLTRAP has shortcomings. Among the issues is the space charge that limits the throughput of the trap. To facilitate the operation of the Penning tap, a MR-TOF (multi-reflection time-of-flight) separator is currently under commissioning at the IGISOL-4. In addition to a pre-separator for JYFLTRAP, the MR-TOF can be used as a stand-alone instrument as will be described in Sect. 4.

In addition to IGISOL, the recoil separators RITU and MARA are utilised in the JYFL-ACCLAB to produce radioactive ion beams. The new vacuum-mode recoil separator MARA (Mass Analysing Recoil Apparatus) [? ] has already proven to be an effective tool for the production of isotopes for research on the proton-rich side of beta stability. Its expansion, MARA-LEB, the low-energy branch of MARA, will utilize the gas cell technique and laser ionization of the recoils stopped and neutralized in flowing noble gas, providing a means to convert $\mathrm{MeV}$-scale radioactive beams to low-energy ones $[17,18]$.

\section{Off-line source array}

The tuning of the beam lines and ion manipulation devices can be performed most conveniently using stable isotope beams from an off-line ion source. In addition, the experiments dealing with atomic masses or other atomic properties quite often can be optimized and tuned using stable isotopes in addition to serving as calibrants. The stable isotope beams have in the past been produced by a glow discharge ion source placed in the IGISOL target chamber at the position of the on-line ion guide. This has not been the most appropriate approach. Already the radiation safety issues related to changing from on-line source to off-line have called for alternative solutions. 
An off-line ion source station with up to three different ion sources has thus been commissioned at IGISOL [19]. Currently, a glow discharge and a heated surface ion source have been installed. The station is located on the top of the radiation shielding of the mass separator area, and is connected to the separator beam line via a vertical beam line. At the bottom of the vertical beam line the ions are bent $90^{\circ}$ towards the dipole magnet by an electrostatic deflector. The bending optics have been designed to accept ions either from the off-line source through the vertical beam line, or from the ion guide in the IGISOL target chamber. Swapping between the sources is implemented by pulsing a steering electrode within the deflector, which allows rapid changing from one beam to another.

The off-line source has already been utilised in several experiments. A variety of stable reference ions with wellknown masses were provided for Penning trap mass measurements at JYFLTRAP [20]. Having the possibility to extract stable isotope beam from two different beamlines allowed measuring the mass difference of stable isobars [21]. A dedicated off-line source afforded the production of a doubly-charged ${ }^{89} \mathrm{Y}$ beam to be used as a reference for laser spectroscopy of doubly-charged fission fragments of yttrium [22].

\section{Hot cavity laser ion source development}

While the bulk of the experiments at IGISOL utilize fission as the production mechanism, the facility also utilizes heavy-ion fusion-evaporation and, more recently, multinucleon-transfer reactions. In recent years, high-precision mass measurements at the JYFLTRAP Penning trap mass spectrometer have been performed using HIGISOL, reaching nuclei with $N=Z-2$ in the region of Tc to $\mathrm{Pd}$ [6].

The ion-guide technique is a fast and universal production mechanism for the production of low-energy beams, however in some cases it suffers from a low efficiency. In order to access specific elements with high efficiency, a new recoil catcher device has been commissioned at IGISOL. The hot cavity catcher laser ion source has been constructed for the production of neutron-deficient silver isotopes due to the particularly beneficial extraction characteristics of silver from graphite [23], which is used as the catcher material [8]. This catcher utilizes inductive heating in order to reach high temperatures exceeding $1700 \mathrm{~K}$, a requirement for fast diffusion of silver out of the catcher material.

Furthermore, as silver is not readily surface ionized, the system utilizes resonance laser ionization as the source of ions. Following the diffusion from the catcher, the atoms effuse into a resistively-heated graphite transfer tube where they are resonantly ionized with counter propagating laser beams. A DC-field resulting from the resistive heating ensures effective and fast, few tens of ms, extraction of the ions. The operation of the catcher system has been demonstrated with in-source laser spectroscopy of neutron-deficient silver isotope using the ${ }^{92 / n a t} \operatorname{Mo}\left({ }^{14} \mathrm{~N}, 2 p x n\right){ }^{104-96} \mathrm{Ag}$ reaction. In the near future the device will be used for the production of even more exotic silver isotopes towards the $N=Z$ line, namely ${ }^{95,96} \mathrm{Ag}$.

\section{MR-TOF separator}

The JYFLTRAP Penning trap setup is used to enhance the achieved mass separation resolution at IGISOL-4. These measurements can be placed in two categories: filter mode and analysis mode measurement. In the filter mode, the trap is used as a high-resolution mass filter that allows only a narrow mass band of ions to pass. Behind the trap, at the post-trap decay spectroscopy station, ions are impinged in the counting position of the spectroscopy set-up. The filter mode is used for post-trap spectroscopy to form a monoisotopic source of radioactive species. Of the spectroscopy experiments benefiting from this kind of sources, the Total Absorbtion Gamma Spectroscopy (TAGS) [24] is of particular note. In TAGS spectroscopy, the only means to distinguish the background events is to subtract them on the basis of spectrum analysis.

In the analysis mode, the ions are detected with a Multichannel Plate (MCP) detector. All ions are counted, within the constraint of the MCP efficiency. The analysis mode is used for atomic mass studies and reaction yield measurements [2, 25].

In the filter mode the priority is the throughput. The main limiting factor is the space charge, the amount of ions that can be simultaneously trapped without severely reducing the trap performance. Since neither the dipole magnet nor RFQ remove the isobaric contaminants, those can be much more abundant than the isotope of interest. The isobaric background is particularly notable when particleinduced fission is used as a production reaction.

In the analysis mode the precision is prioritised. This means that the count rate is kept very low: in precise atomic mass measurements a single ion in the precision trap at a time is preferred. However, if the ratio of isotope of interest to the isobaric background is orders of magnitude in favour of the isobars, the resolution of the purification trap is lost, even if the absolute space charge limit is not reached.

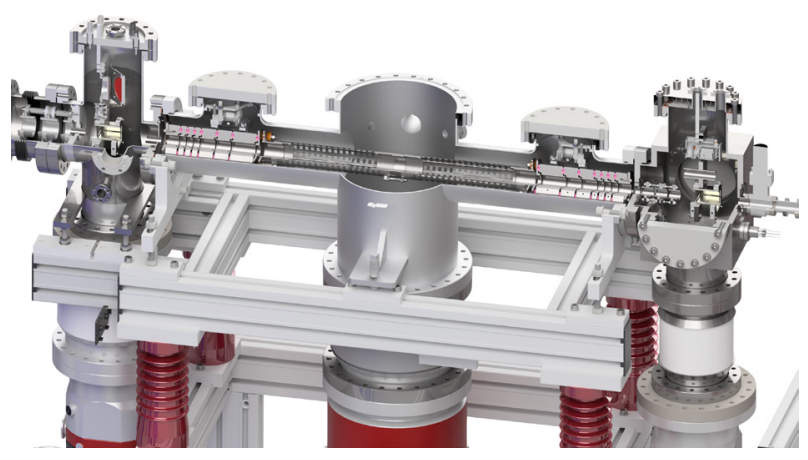

Figure 2. Cut-away wiew of the MR-TOF separator/spectrometer. During the separation, the ions are moving back and forth between electrostatic mirrors. A mass resolution of $R \approx 37000$ was reached off-line in $\sim 2.6 \mathrm{~ms}$ or 83 oscillations. 


\subsection{MR-TOF as pre-separator for JYFLTRAP}

In both aforementioned modes, the JYFLTRAP Penning trap performance can be improved by removing the isobaric contaminants from the beam entering the Penning trap. To this extent, a Multi-Reflection Time-Of-Flight (MR-TOF) separator/spectrometer has recently been installed in the beamline before the Penning trap, see Fig. 3.

The MR-TOF separator consists of two electrostatic mirrors and a drift tube in between (Fig 2). The ions are trapped between the mirrors by lowering the drift tube potential with respect to mirrors, and extracted at the end by lifting the potential. The reflection is energy-isochronous: all ions with the same energy turn around in the same time. The time-of-flight of the ions thus depends linearly on their mass, as well as on the number of oscillations between the mirrors. In preliminary off-line tests, a mass resolving power of $R \approx 37000$ was reached in $\sim 2.6 \mathrm{~ms}$ or 83 oscillations with stable ${ }^{85} \mathrm{Rb}$ ions. Such a resolution is already sufficient to resolve most of the isobaric contamination. In particular, in studies of neutron-rich nuclei produced in fission, the isotope of interest is often separated by several $\mathrm{MeV}$ from the nearest contaminant, requiring only $R \approx 10^{4} \ldots 10^{5}$ mass resolving power.

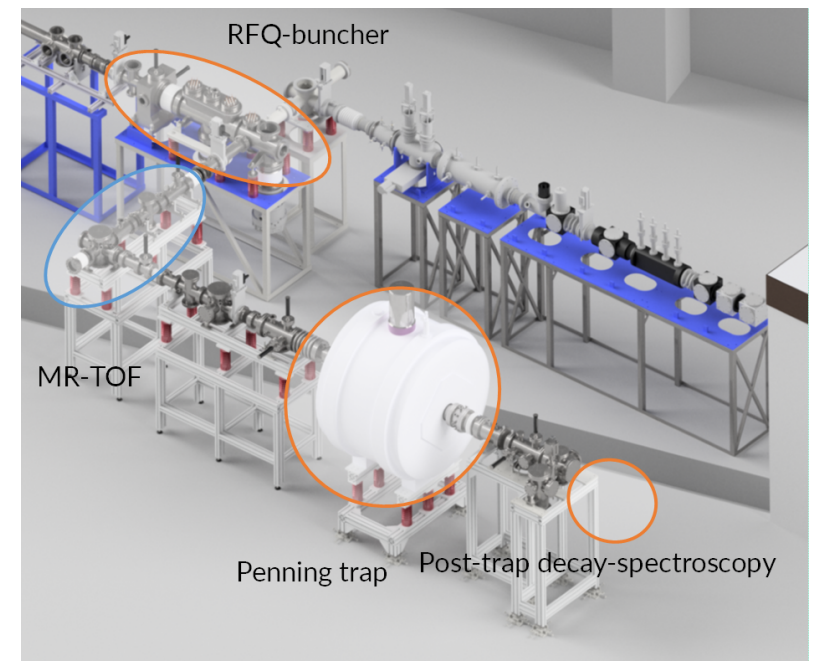

Figure 3. The MR-TOF separator/spectrometer is placed in the beamline between the RFQ and JYFLTRAP. The bends of the beamline are realised with quadrupole benders. Thus, instead of turning left after the MR-TOF, the beam can be delivered to an experimental setup straight ahead or to the right.

\subsection{Stand-alone separator operation}

Instead of delivering the separated ion bunch to JYFLTRAP for analysis or further purification, the selected ion bunch can be guided directly to the counting position of a spectroscopy setup. Reaching sufficient purification with the MR-TOF is over an order of magnitude faster than with JYFLTRAP.

The execution of many spectroscopy experiments without isobaric purification in the spectroscopy line ("6" in Fig 1) are limited due to the detector system count rate caused by the decay of the isobaric contaminants; not due to the yield from the IGISOL. Most decay-spectroscopy experiments being carried out at IGISOL are studies of rather exotic nuclei. As the mass difference between the nuclei of interest and the nearby isobaric contamination is rather large (several $\mathrm{MeV} / \mathrm{c}^{2}$ ), it is in most cases enough to utilize only the MR-TOF without the need for additional purification with JYFLTRAP. Cases requiring $R \approx 10^{5}$ are sufficiently separated with the MR-TOF.

\subsection{Yield measurements with MR-TOF}

An MR-TOF is extremely suitable for yield measurements. Unlike the Penning trap, which requires frequency scanning, an MR-TOF does not require any parameter scanning as the ions are separated by their time-of-flight. A typical example of a yield measurement are the isotopic yields in fission reactions [2]. Such a measurement with a Penning trap requires typically 50 frequency points to be scanned with several tens of rounds of scanning; one scan point taking up to $500 \mathrm{~ms}$ to complete. With an MR-TOF, the same scan be achieved in a small fraction of that time.

\section{Phase-dependent cleaning method}

The mass resolving power of JYFLTRAP in the filter mode depends on the utilised technique. Currently, there are three techniques available. With the purification trap, using the sideband cooling technique [26], a resolution of $R \sim 10^{5}$ can be routinely reached. Far from stability, where the mass difference between adjacent isotopes within the isobar is of the order of several $\mathrm{MeV} / c^{2}$, much lower resolution is usually sufficient to separate different isotopes [14]. A second method, the Ramsey cleaning method in the precision trap, is capable of separating masses with about an order of magnitude better resolving power. With this method it is possible to resolve many isomeric states from the nuclear ground state. A mass resolving power of the order of $R \sim 10^{6}$ has been reached, allowing separation of states with a few $100 \mathrm{keV} / \mathrm{c}^{2}$ mass difference [15].

The third and the most recent technique involves utilization of ion motional phases in the trap [13]. The method is very similar to the PI-ICR mass measurement technique [27]. The separation is achieved by accumulating a large enough phase difference in cyclotron motion. Given enough time, up to a few seconds even, a mass resolving power up to $10^{7}$ is available. Since the ions are only spatially separated, there is a need to use a downstream collimator or mechanical slits to remove the unwanted state(s).

The biggest challenge in using a Penning trap as a mass filter is the space charge limitation. The higher the resolving power, the more severe the limitation.

\section{MARA Low-Energy Branch}

The new vacuum-mode recoil separator MARA [? ] is an effective tool for research on the proton-rich nuclei. The 
low-energy branch of MARA, MARA-LEB, is being constructed at the JYFL-ACCLAB to further expand the opportunities of MARA. Its purpose is to enable laser spectroscopy and atomic mass measurements in the vicinity of $N=Z$ line in the mass range $79<A<108$. Of particular interest are the neutron-deficient $\mathrm{Zr}, \mathrm{Ag}$ and $\mathrm{Sn}$ isotopes [17].

In the MARA-LEB concept, the neutron-deficient heavy-ion fusion reaction recoils are separated with MARA and stopped in a gas cell located at the focal plane of thr separator. In contrast to ion guide technique, the ions are neutralised and transported via the gas flow out of the gas cell. At the exit of the cell, a supersonic gas jet carrying the neutral atoms is formed. The atoms can be selectively laser-ionised in the gas cell or gas jet, accelerated and transported for subsequent mass analysis. In this way the possible contaminants with the same $m / q$ are removed. More important however is that the energy of the reaction products of interest is reduced from $\mathrm{MeV}$ scale to $\mathrm{keV}$ energies, making low-energy ground state measurements via optical spectroscopy and mass spectrometry feasible. In a later phase of MARA-LEB, an RFQ cooler and buncher will be introduced, reducing the achievable ion energies to the $\mathrm{eV}$ scale. In addition, a similar MR-TOF spectrometer as currently being commissioned at the IGISOL-4 will be installed for atomic mass measurements $[17,18]$.

Currently, the development and construction of various parts of MARA-LEB is underway. The gas cell has been designed and built, and tests with an alpha-recoil source are in progress. Pulsed Ti:sapphire lasers, vacuum and gas purification systems have been built and are under testing. The main items for the mass analysis and transfer line have been purchased. Ion-optical simulations of the transfer line and ion guides have been verified, the mechanical design is ongoing and expected to be finalised for the most part during 2019.

\section{References}

[1] I. Moore, T. Eronen, D. Gorelov, J. Hakala, A. Jokinen, A. Kankainen, V. Kolhinen, J. Koponen, H. Penttilä, I. Pohjalainen et al., Nuclear Instruments and Methods in Physics Research Section B: Beam Interactions with Materials and Atoms 317, 208 (2013)

[2] H. Pronttilä, D. Gorelov, V.V. Elomaa, T. Eronen, U. Hager, J. Hakala, A. Jokinen, A. Kankainen, P. Karvonen, I.D. Moore et al., The European Physical Journal A 52, 104 (2016)

[3] M. Fadil, M. Lewitowicz, L. Fortin, N. Ménard, Interactive charts of beams of GANIL and European facilities, EPS Divisional Conference: Towards EURISOL Distributed Facility (2016), poster, http://hal . in2p3. fr/in2p3-01569720

[4] T. Eronen, J.C. Hardy, L. Canete, A. Jokinen, J. Hakala, A. Kankainen, V.S. Kolhinen, J. Koponen, I.D. Moore, I.M. Murray et al., Physical Review C 95, 025501 (2017)

[5] P. Dendooven, R. Béraud, E. Chabanat, A. Emsallem, A. Honkanen, M. Huhta, A. Jokinen,
G. Lhersonneau, M. Oinonen, H. Penttilä et al., Nuclear Instruments and Methods in Physics Research A: Accelerators, Spectrometers, Detectors and Associated Equipment 408, 530 (1998)

[6] C. Weber, V.V. Elomaa, R. Ferrer, C. Fröhlich, D. Ackermann, J. Äystö, G. Audi, L. Batist, K. Blaum, M. Block et al., Physical Review C 78, 054310 (2008)

[7] K. Peräjärvi, J. Cerny, J. Hakala, J. Huikari, A. Jokinen, P. Karvonen, J. Kurpeta, D. Lee, I. Moore, H. Penttilä et al., Nuclear Instruments and Methods in Physics Research Section A: Accelerators, Spectrometers, Detectors and Associated Equipment 546, 418 (2005)

[8] M. Reponen, I.D. Moore, I. Pohjalainen, S. Rothe, M. Savonen, V. Sonnenschein, A. Voss, Review of Scientific Instruments 86, 123501 (2015)

[9] P. Karvonen, I. Moore, T. Sonoda, T. Kessler, H. Penttilä, K. Peräjärvi, P. Ronkanen, J. Äystö, Nuclear Instruments and Methods in Physics Research Section B: Beam Interactions with Materials and Atoms 266, 4794 (2008)

[10] A. Nieminen, J. Huikari, A. Jokinen, J. Äystö, P. Campbell, E. Cochrane, Nuclear Instruments and Methods in Physics Research Section A: Accelerators, Spectrometers, Detectors and Associated Equipment 469, 244 (2001)

[11] R. [de Groote], A. [de Roubin], P. Campbell, B. Cheal, C. Devlin, T. Eronen, S. Geldhof, I. Moore, M. Reponen, S. Rinta-Antila et al., Nuclear Instruments and Methods in Physics Research Section B: Beam Interactions with Materials and Atoms 463, 437 (2020)

[12] T. Eronen, V.V. Elomaa, U. Hager, J. Hakala, A. Jokinen, A. Kankainen, S. Rahaman, J. Rissanen, C. Weber, J. Äystö, Nuclear Instruments and Methods in Physics Research Section B: Beam Interactions with Materials and Atoms 266, 4527 (2008), proceedings of the XVth International Conference on Electromagnetic Isotope Separators and Techniques Related to their Applications

[13] D.A. Nesterenko, T. Eronen, A. Kankainen, L. Canete, A. Jokinen, I.D. Moore, H. Penttilä, S. Rinta-Antila, A. de Roubin, M. Vilen, The European Physical Journal A 54, 154 (2018)

[14] T. Eronen, V.S. Kolhinen, V.V. Elomaa, D. Gorelov, U. Hager, J. Hakala, A. Jokinen, A. Kankainen, P. Karvonen, S. Kopecky et al., The European Physical Journal A 48, 46 (2012)

[15] K. Peräjärvi, T. Eronen, A. Cagniant, D. Gorelov, J. Hakala, A. Jokinen, A. Kankainen, H. Kettunen, V. Kolhinen, M. Laitinen et al., Applied Radiation and Isotopes 71, 34 (2013)

[16] J. Uusitalo, J. Sarén, J. Partanen, J. Hilton, Acta Physica Polonica B 50, 319 (2019)

[17] P. Papadakis, I. Moore, I. Pohjalainen, J. Sarén, J. Uusitalo, Hyperfine Interactions 237, 152 (2016) 
[18] P. Papadakis, I. Moore, T. Eronen, J. Liimatainen, T. Kalvas, J. Partanen, I. Pohjalainen, M. Reponen, S. Rinta-Antila, J. Sarén et al., AIP Conference Proceedings 2011, 070013 (2018)

[19] M. Vilén, L. Canete, B. Cheal, A. Giatzoglou, R. [de Groote], A. [de Roubin], T. Eronen, S. Geldhof, A. Jokinen, A. Kankainen et al., Nuclear Instruments and Methods in Physics Research Section B: Beam Interactions with Materials and Atoms 463, 382 (2020)

[20] M. Vilen, J.M. Kelly, A. Kankainen, M. Brodeur, A. Aprahamian, L. Canete, R.P. de Groote, A. de Roubin, T. Eronen, A. Jokinen et al., Physical Review C 101, 034312 (2020)

[21] D. Nesterenko, L. Canete, T. Eronen, A. Jokinen, A. Kankainen, Y. Novikov, S. Rinta-Antila, A. de Roubin, M. Vilen, International Journal of Mass Spectrometry 435, 204 (2019)

[22] L.J. Vormawah, M. Vilén, R. Beerwerth, P. Campbell, B. Cheal, A. Dicker, T. Eronen, S. Fritzsche,
S. Geldhof, A. Jokinen et al., Physical Review A 97, 042504 (2018)

[23] R. Kirchner, Nuclear Instruments and Methods in Physics Research Section B: Beam Interactions with Materials and Atoms 70, 186 (1992)

[24] V. Guadilla, A. Algora, J.L. Tain, M. Estienne, M. Fallot, A.A. Sonzogni, J. Agramunt, J. Äystö, J.A. Briz, A. Cucoanes et al., Physical Review Letters 122, 042502 (2019)

[25] T. Eronen, A. Kankainen, J. Äystö, Progress in Particle and Nuclear Physics 91, 259 (2016)

[26] G. Savard, S. Becker, G. Bollen, H.J. Kluge, R. Moore, T. Otto, L. Schweikhard, H. Stolzenberg, U. Wiess, Physics Letters A 158, 247 (1991)

[27] S. Eliseev, K. Blaum, M. Block, C. Droese, M. Goncharov, E. Minaya Ramirez, D.A. Nesterenko, Y.N. Novikov, L. Schweikhard, Physical Review Letters 110, 082501 (2013) 